\title{
Upper Gastrointestinal Bleeding as the First Manifestation of Wegener's Granulomatosis
}

\author{
Hamid Tavakkoli ${ }^{1}$, Mehdi Zobeiri ${ }^{*}$, Mansour Salesi ${ }^{3}$, Mohammad Hossein Sanei ${ }^{4}$
}

1. Department of Gastroenterology, AlZahra Hospital, School of Medicine, Isfahan University of Medical Sciences, Isfahan, Iran

2. Department of Internal Medicine, Imam Reza Hospital, , School of Medicine, Kermanshah University of Medical Sciences, Kermanshah, Iran

3. Department of Rheumatology, Al-Zahra Hospital, School of Medicine, Isfahan University of Medical Sciences, Isfahan, Iran

4. Department of pathology, Al-Zahra Hospital, School of Medicine, Isfahan University of Medical Sciences, Isfahan, Iran

* Corresponding Author:

Mehdi Zobeiri, MD

Zacaria Razi Boulevard, Imam Reza

Hospital, Kermanshah, Iran

Post code: 6714415333

Tel: + 989188311726

Fax: +988314276343

Email: mzoberi@KUMS.ac.ir

Received: 28 Feb. 2016

Accepted: 10 May 2016

\section{ABSTRACT}

Wegener's granulomatosis is an uncommon inflammatory disease that manifests as vasculitis, granulomatosis, and necrosis. It usually involves the upper and lower respiratory tracts and kidneys. Although it may essentially involve any organ, gastrointestinal (GI) involvement is notably uncommon.

A 20-year-old male patient presented with epigastric pain, vomiting, hematemesis, and melena. On physical examination, he was pale. There was no abdominal tenderness or organomegaly. Upper GI endoscopy revealed dark blue-colored infiltrative lesions in prepyloric area. Evaluation of the biopsy sample showed mononuclear cell infiltration in the submucosal area, hyperplastic polyp, and chronic gastritis. High dose proton pump inhibitor and adjunctive supportive measures were given but no change in the follow-up endoscopy was detected. During hospital course, he developed intermittent fever and serum creatinine elevation. 12 days after admission, he developed dyspnea, tachypnea, and painful swelling of metacarpophalangeal joints, and maculopapular rash in extensor surface of the right forearm. Chest radiography showed pulmonary infiltration. Serum c-ANCA titer was strongly positive and skin biopsy revealed leukocytoclastic vasculitis. The patient received methylprednisolone pulse, which resulted in complete recovery of symptoms and gastric lesion.

The present case indicates that GI bleeding may be the first manifestation of Wegener's granulomatosis. Moreover, it should be emphasized that gastric biopsy is not characteristic or diagnostic in such patients.

\section{KEYWORDS}

Wegener's granulomatosis; Gastrointestinal symptoms, Vasculitis, ANCA.

Please cite this paper as:

Tavakkoli H, Zobeiri M, Salesi M, Sanei MH. Upper Gastrointestinal Bleeding as the First Manifestation of Wegener's Granulomatosis. Middle East J Dig Dis 2016;8:235-239. DOI $: 10.15171 /$ mejdd.2016.27

\section{INTRODUCTION}

Wegener's Granulomatosis (WG), also known as granulomatosis with polyangiitis is a systemic small-vessel vasculitides associated with anti-neutrophil cytoplasmic antibodies (ANCA). ${ }^{1}$ WG is an uncommon and severe granulomatous necrotizing vasculitis, which affects the upper and lower respiratory tracts and kidneys. ${ }^{2}$ It can present with multiple pulmonary and renal lesions, including pulmonary nodule, cavitary lung lesions, fibrosis, and a rapidly progressive crescentic glomerulonephritis of the kidney. ${ }^{1,}{ }^{3}$

Gastrointestinal (GI) manifestations of WG are uncommon and gastric in- 

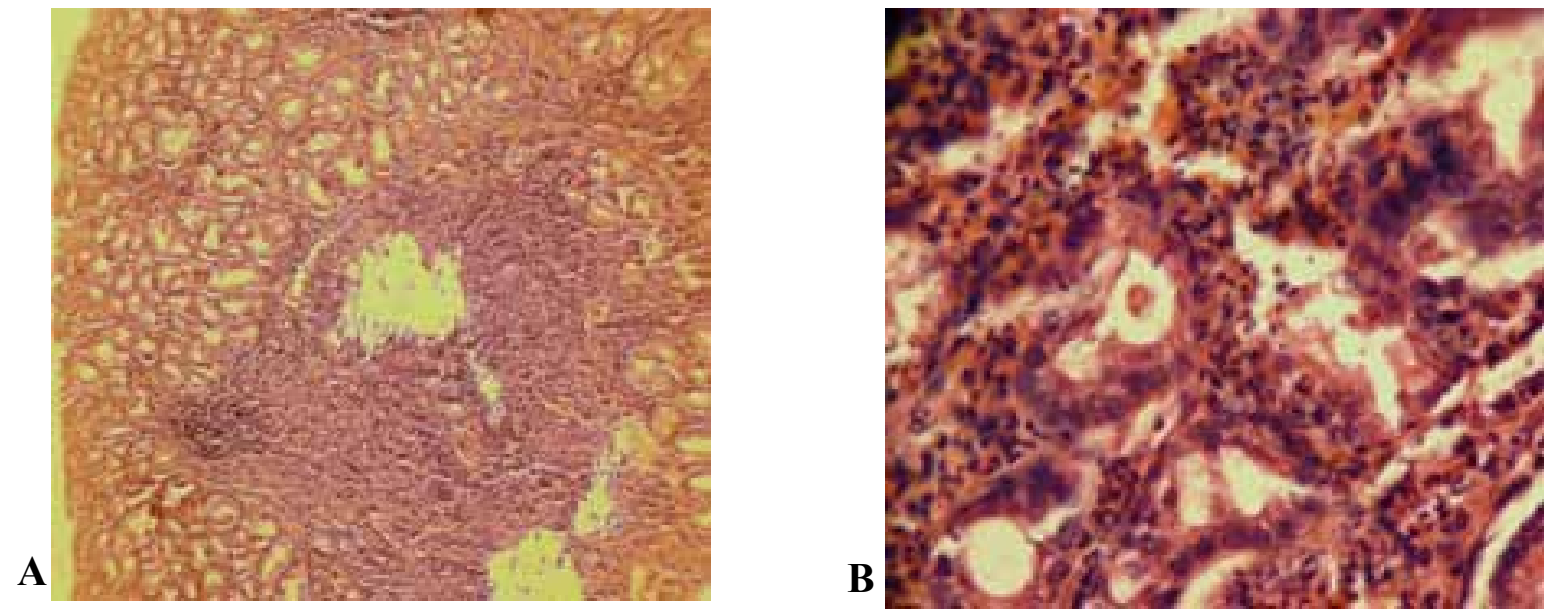

Fig.1: Gastric biopsy (H \& E staining): A) low magnification (X 100) shows focal gastritis. B) High magnification (X 400) reveals active chronic gastritis.

volvement is rare and non-specific. A review of literature showed only two cases in whom gastric involvement, as massive and frequent gastric hemorrhages were the presenting features..$^{4-6}$

\section{CASE REPORT}

A 20-year-old white male patient presented with epigastric pain, vomiting, hematemesis, and melena since 10 days prior to the admission. He had the history of recurrent sinusitis and frequent ibuprofen consumption. There was no other significant medical history and he was not an active smoker. He was conscious with stable vital signs, had nasal speech and no scleral icterus. Examination of neck, ears, and nose showed purulent post-nasal discharge. Heart, lung, and abdominal examinations were not remarkable. Upper GI endoscopy revealed blue black colored necrotic infiltrative lesions in prepyloric area (about $5 \mathrm{~cm}$ long). The biopsy revealed focal mononuclear cell infiltration in the mucosal area associated with active gastritis. There was no vasculitis or granuloma and also no organism was identified on Giemsa staining (figure 1).

High dose proton pump inhibitor and adjunctive supportive measures were given but the patient had recurrent hematemesis. No visible changes in repeated endoscopies were detected. During the hospital course, he developed intermittent fever and elevation of serum creatinine. Serum hemoglobin level demonstrated decreasing pattern and two units of packed cells were trans- fused. In 12 days after admission, dyspnea-tachypnea, and painful swelling of metacarpophalangeal joints, and maculopapular rash in extensor surface of the right forearm were developed.

On physical examination, pericardial friction rub, bilateral decreased breathing sounds, and basilar fine crackle were detected. Chest radiography and high resolution computed tomography of the lung revealed high normal heart shadow, bilateral basilar alveolar opacities, and bilateral pleural effusions (figure 2). Echocardiography revealed pericardial effusion. Urinalysis revealed many dysmorphic RBCs without RBC casts and 8-10 WBCs/hpf. Serum creatinine level rose from 1 to 3.3 $\mathrm{mg} / \mathrm{dL}$. Leukocytosis (20700) with PMN dominancy was detected. After taking appropriate culture specimen, antibiotic was administered without any response. Other laboratory investigations showed the following data: serologic tests for hepatitis $\mathrm{B}$, hepatitis $\mathrm{C}$, and human immunodeficiency viruses showed negative results, electrolyte profile, and antinuclear antibodies were normal, C-reactive protein was $2+$, and serum c-ANCA (antineutrophil cytoplasmic antibody) titer was strongly positive (455.5). A biopsy of skin revealed leukocytoclastic vasculitis.

According to clinical course and mentioned paraclinical findings diagnosis of WG was made on and treatment with methylprednisolone pulse $1000 \mathrm{mg}$ daily was started. The patient showed clinical improvement and gradual disease resolution. Five days later, upper GI endoscopy 

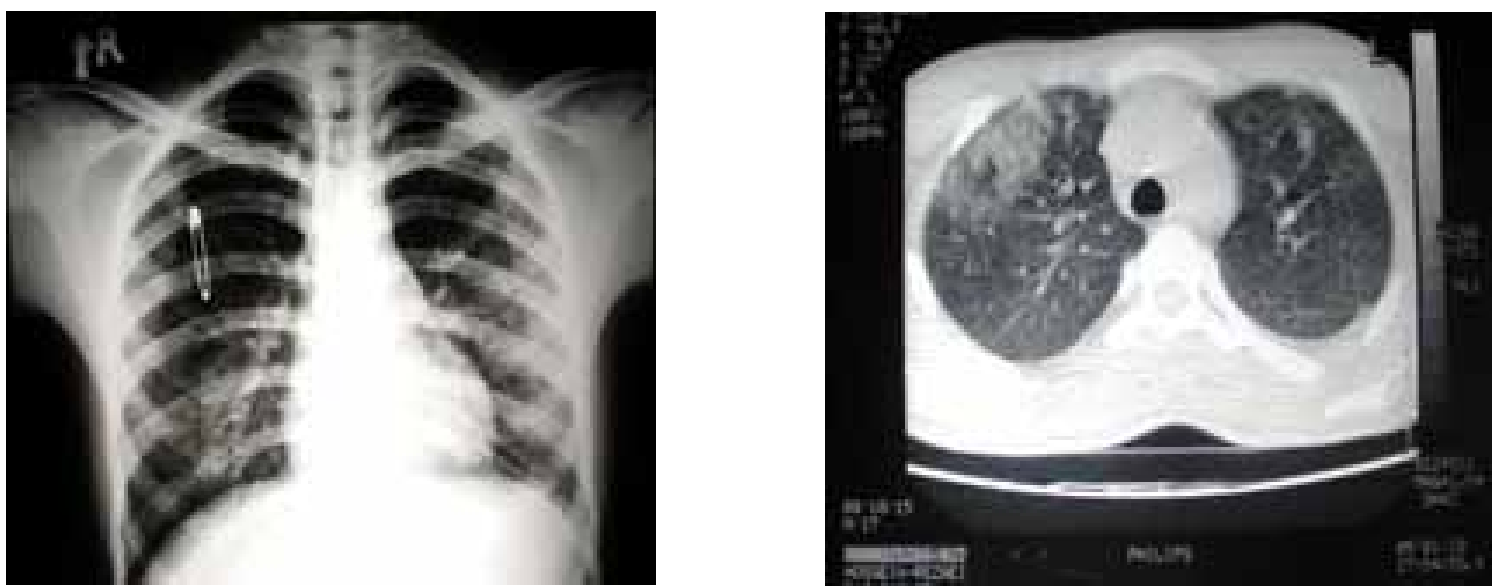

Fig.2: Chest radiograph and high resolution computed tomogram of lung showing pulmonary infiltrations.

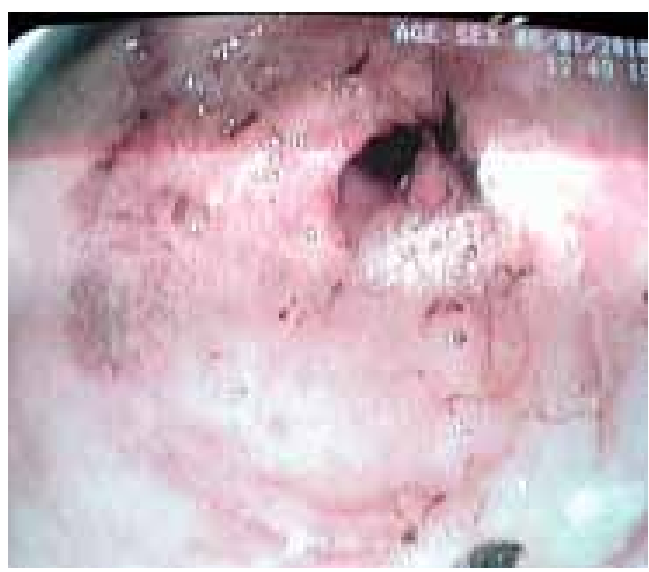

Fig.3: Upper gastrointestinal endoscopy showing antral and prepyloric lesions five days after treatment

showed significant improvement (figure 3). Treatment was continued by prescribing cyclophosphamide monthly pulse. Gastric lesion was completely healed and GI bleeding did not recur after 6 months follow-up. All pulmonary, renal, articular, and skin lesions were recovered.

\section{DISCUSSION}

Most patients with WG show multisystemic disease in clinical course. ${ }^{7}$ However, GI involvement is very uncommon and is usually detected in autopsy studies. ${ }^{8}$ Involvement of the GI tract in WG is relatively rare and usually occurs several years after the onset of initial symptoms and its treatment. ${ }^{9,10}$ A large prospective study of 158 patients with WG showed no gastrointestinal involvement throughout the course of disease. ${ }^{1}$ The small bowel is the most common site involved by
WG. ${ }^{12,13}$ Clinically, gastrointestinal WG mimics inflammatory and infectious bowel diseases. ${ }^{14} \mathrm{WG}$ may mimic Crohn's disease with granulomatous gastritis or ileitis, ${ }^{15,16}$ Inflammatory ileocolitis with hemorrhage, gangrenous cholecystitis, and bowel infarction, which have been reported earlier. ${ }^{17}$

Furthermore, three patients with esophageal involvement were reported. A patient whose initial presentation of WG was odynophagia secondary to esophageal vasculitis was described in whom multiple punched out ulcerations in the esophagus were detected, which resolved by standard therapy for systemic WG. ${ }^{18}$

In 1990, the American College of Rheumatology (ACR) established the criteria for the classification of WG as follows: (1) Nasal or oral manifestation (painful or painless oral ulcers or purulent or bloody nasal discharge) (2) Abnormal Chest radiography showing nodules, fixed infiltrates, or cavities (3) Abnormal urinary sediment (microscopic hematuria with or without red cell casts) and (4) Granulomatous inflammation on biopsy of an artery or perivascular area. Patients should have at least 2 of these 4 criteria to be labeled as having WG. ${ }^{19}$

The diagnosis of WG was based on the clinical manifestations, imaging studies showing progressive multisystem involvements especially renal, and upper and lower respiratory tracts, skin biopsy, and presence of high titer of c-ANCA. The diagnosis was further supported in our patient by mild leukocytosis and normocytic normochromic anemia. Presence of c-ANCA or PR3-ANCA by immunofluorescence is recognized as a 
reliable and valuable diagnostic tool with high specificity in the absence of histopathological evaluation for the diagnosis of WG and is extremely helpful in differentiating WG from other diseases. ${ }^{20}$ Occasionally, patients with infections, neoplasms, inflammatory bowel disease, sclerosing cholangitis, and other rheumatologic diseases develop ANCA, but these are predominantly perinuclear ANCAs (p-ANCA) or exhibit an atypical staining pattern. $^{21}$

Our patient had a long history of recurrent sinusitis, which may represent a slowly progressive mild WG. He fulfilled three ACR criteria for diagnosis of WG. In addition, the diagnosis was further supported by mild leukocytosis and normocytic normochromic anemia and high titer of C-ANCA.

The histological proof of necrotizing vasculitis is dependent on the depth of the biopsy and therefore, can be easily missed. ${ }^{22}$ In our patient, stomach lesion appearance was not typical for peptic ulcer or tumoral mass. It was a blue black necrotic lesion and the biopsy was not diagnostic. Treatment was initiated with pulse of steroid and cyclophosphamide, according to the clinical course and laboratory findings especially high titer of c-ANCA. Response to treatment was achieved in short-term. Immunosuppressive therapy combined with routine antiulcer treatment has been shown very effective in repairing ulcerative lesions. ${ }^{23}$ Application of aggressive immunotherapy in this disease is justified because survival in patients with untreated WG is extremely poor. ${ }^{24}$

In conclusion, upper GI bleeding may be the first presenting feature of WG. Considering this fact can lead to an early diagnosis and treatment, thereby to avoid unnecessary surgery and potential mortality.

\section{CONFLICT OF INTEREST}

The authors declare no conflict of interest related to this work.

\section{REFERENCES}

1. Mahr A, Katsahian S, Varet H, Guillevin L, Hagen EC, Hoglund $\mathrm{P}$, et al. Revisiting the classification of clinical phenotypes of anti-neutrophil cytoplasmic antibody-associated vasculitis: a cluster analysis. Ann Rheum Dis 2013;72:100310. doi: 10.1136/annrheumdis-2012-201750.

2. Frankel SK, Cosgrove GP, Fischer A, Meehan RT, Brown
KK. Update in the Diagnosis and Management of Pulmonary Vasculitis. Chest 2006;129:452-65. doi:10.1378/ chest.129.2.452

3. Shahedi K, Hanna RM, Melamed O, Wilson J. Wegener's granulomatosis mimicking inflammatory bowel disease and presenting with chronic enteritis. Int Med Case Rep J 2013;6:65-9. doi: 10.2147/IMCRJ.S36546.

4. Storesund B, Gran JT, Koldingsnes W. Severe intestinal involvement in Wegener's granulomatosis: report of two cases and review of the literature. Br J Rheumatol 1998;37:387- 90. doi: 10.1093/rheumatology/37.4.387

5. Deger SM, Sahin T, Vural C, Aygencel G, Kerem M, Onal $\mathrm{B}$, Tezel E. Wegener's granulomatosis with massive gastrointestinal hemorrhage due to jejunal and colonic involvement: report of a case. Surg Today 2008;38:166-9. doi: 10.1007/s00595-007-3574-9.

6. Yamauchi F, Tanabe R, Nakashima R, Endoh M, Nomoto $\mathrm{Y}$, Sakai H, et al. Wegener's granulomatosis with relapsed bleeding of gastric ulcers: a case report.Tokai J Exp Clin Med 1995;20:233-40.

7. Hoffman GS, Kerr GS, Leavitt RY Hallahan CW, Lebovics RS, Travis WD, et al. Wegener granulomatosis: an analysis of 158 patients. Ann Intern Med 1992;116:488-98.

8. Watts RA, Scott DG. Epidemiology of the vasculitides. Semin Respr Crit Care Med 2004;25:455-64.

9. Qian Q, Cornell L, Chandan V, Hartman R, Caples S. Hemorrhagic Colitis as a Presenting Feature of Wegener Granulomatosis. J Gastrointestin Liver Dis 2010;19:445-7.

10. Steele C, Bohra S, Broe P, Murray FE. Acute upper gastrointestinal haemorrhage and colitis: an unusual presentation of Wegener's granulomatosis. Eur J Gastroenterol Hepatol 2001;13:993-5.

11. Akca T, Colak T, Caglikulekci M, Ocal K, Aydin S. Intestinal perforation in Wegener's granulomatosis: a case report. Ulus Travma Derg 2005;11:348-51.

12. Storesund B, Gran JT, Koldingsnes W. Severe intestinal involvement in Wegener's granulomatosis: report of two cases and review of the literature. $\mathrm{Br} J$ Rheumatol 1998;37:387- 90. doi: 10.1093/rheumatology/37.4.387

13. Chow FY, Hooke D, Kerr PG. Severe intestinal involvement in Wegener's granulomatosis. J Gastroenterol Hepatol 2003;18:749-750. doi: 10.1046/j.1440-1746.2003.30472.x

14. Veinot JP, Logan CA, Thomas MJ. Wegener's granulomatosis arteritis causing small bowel infarction. Pathology 2003;35:268-9. doi:10.1080/0031302031000151046

15. Temmesfeld-Wollbrueck B, Heinrichs C, Szalay A, Seeger W. Granulomatous gastritis in Wegener's disease: Differentiation from Crohn's disease supported by a positive test for antineutrophil antibodies. Gut 1997;40:550. doi:10.1136/gut.40.4.550

16. Sokol R, Farrell M, McAdams A. An unusual presentation of Wegener's granulomatosis mimicking inflammatory bowel disease. Gastroenterology 1984;87:426-32. 
17. Storesund B, Gran J, Koldingsnes W. Severe intestinal involvement in Wegener's granulomatosis: Report of two cases and review of the literature. Rheumatology 1998;37:387-90. doi: 10.1093/rheumatology/37.4.387

18. Fallows GA, Hamilton SF, Taylor DS, Reddy SB. Esophageal involvement in Wegener's granulomatosis: a case report and review of the literature, Can J Gastroenterol 2000;14:449-51. doi:10.1155/2000/423569.

19. Leavitt RY, Fauci AS, Bloch DA, Michel BA, Hunder GG, Arend WP, et al, The American College of Rheumatology 1990 criteria for the classification of wegener's granulomatosis,. Arthritis Rheum 1990;33:1101-7. doi: 10.1002/art.1780330807

20. Bosch X, Guilabert A, Font J. Antineutrophil cytoplasmic antibodies. Lancet 2006;368:404-18. doi: 10.1002/ art.1780330807

21. Gross WL. Antineutrophil cytoplasmic autoantibody testing in vasculitides. Rheum Dis Clin North Am 1995;21:9871011.

22. Yamauchi F, Tanabe R, Nakashima R, Endo M, Nomoto $\mathrm{Y}$, et al. Wegner's granulomatosis with relapsed bleeding of gastric ulcers,: a case report, Tokai J Exp Clin Med, 1995;20:233-40. doi: 10.3122/jabfm.16.6.555

23. RadjenovicT, Radovic P, Pejcic T, Nastasijevic D, Djordjevic I. Wegener's granulomatosis: Case Report. Acta Medica Medianae 2008;47:78-81.

24. Wegener Granulomatosis: Case Report and Brief Literature Review. J Am Board Fam Pract 2003;16:555-9. doi: 10.3122/jabfm.16.6.555 